\title{
Triplets of Closely Embedded Dirichlet Type Spaces on the Unit Polydisc
}

\author{
Petru Cojuhari · Aurelian Gheondea
}

Received: 23 July 2012 / Accepted: 8 October 2012 / Published online: 27 October 2012 (C) Springer Basel 2012

\begin{abstract}
We propose a general concept of triplet of Hilbert spaces with closed embeddings, instead of continuous ones, and we show how rather general weighted $L^{2}$ spaces yield this kind of generalized triplets of Hilbert spaces for which the underlying spaces and operators can be explicitly calculated. Then we show that generalized triplets of Hilbert spaces with closed embeddings can be naturally associated to any pair of Dirichlet type spaces $\mathcal{D}_{\alpha}\left(\mathbb{D}^{N}\right)$ of holomorphic functions on the unit polydisc $\mathbb{D}^{N}$ and we explicitly calculate the associated operators in terms of reproducing kernels and radial derivative operators. We also point out a rigging of the Hardy space $H^{2}\left(\mathbb{D}^{N}\right)$ through a scale of Dirichlet type spaces and Bergman type spaces.
\end{abstract}

Keywords Closed embedding - Triplet of Hilbert spaces - Rigged Hilbert spaces · Dirichlet type space on the unit polydisc $\cdot$ Kernel operator $\cdot$ Hamiltonian

Communicated by Daniel Aron Alpay.

P. Cojuhari acknowledges financial support from the Polish Ministry of Science and Higher Education: 11.11.420.04 and Grant NN201 546438 (2010-2013).

\section{P. Cojuhari}

Department of Applied Mathematics, AGH University of Science and Technology, Al. Mickievicza 30, 30-059 Cracow, Poland

e-mail: cojuhari@uci.agh.edu.pl

A. Gheondea $(\bowtie)$

Department of Mathematics, Bilkent University, 06800 Bilkent, Ankara, Turkey e-mail: aurelian@fen.bilkent.edu.tr

A. Gheondea

Institutul de Matematică al Academiei Române, C.P. 1-764, 014700 Bucureşti, România

e-mail: A.Gheondea@imar.ro

Birkhäuser 
Mathematics Subject Classification (1991) 47A70 - 47B25 - 47B34 - 46E22 . $46 \mathrm{E} 20$

\section{Introduction}

Motivated by the necessity of reconciliating the two mathematical formalisms of Quantum Mechanics, that of P.A.M. Dirac based on bras and kets formalism, and that of J. von Neumann based on Hilbert space formalism, and facilitated by the appearance of L. Schwartz's theory of distributions [22], Gelfand and his school, see e.g. [14], introduced the notion of rigged Hilbert spaces. Since 1960's, the rigged Hilbert space formalism was recognized and intensively used by physicists as a powerful and rigorously mathematical tool that reflects most of the aspects of the Dirac's formalism of quantum mechanics, e.g. see [10,19], and the rich bibliography cited there. In this respect, we mention Gadella's work [13] that describes a rigging of $L^{2}(0, \infty)$ through Hardy classes of analytic functions in the open half-planes and its applications to resonances in quantum mechanics.

An important variation of the construction of rigged Hilbert spaces was done by Berzanskii [6] and his school [7,8], through a scale of Hilbert spaces and where the main step is made by the so-called triplet of Hilbert spaces. More precisely, this is denoted by $\mathcal{H}_{+} \hookrightarrow \mathcal{H} \hookrightarrow \mathcal{H}_{-}$, where: $\mathcal{H}_{+}, \mathcal{H}$, and $\mathcal{H}_{-}$are Hilbert spaces, the embeddings are continuous (bounded linear operators), the space $\mathcal{H}_{+}$is dense in $\mathcal{H}$, the space $\mathcal{H}$ is dense in $\mathcal{H}_{-}$, and the space $\mathcal{H}_{-}$is the conjugate dual of $\mathcal{H}_{+}$with respect to $\mathcal{H}$, that is, $\|\varphi\|_{-}=\sup \left\{\left|\langle h, \varphi\rangle_{\mathcal{H}}\right|\|h\|_{+} \leq 1\right\}$, for all $\varphi \in \mathcal{H}$.

Extending these triplets on both sides, one gets a scale of Hilbert spaces

$$
\begin{aligned}
\cdots \hookrightarrow \mathcal{H}_{+n} \hookrightarrow \mathcal{H}_{+(n-1)} & \hookrightarrow \cdots \hookrightarrow \mathcal{H}_{+1} \hookrightarrow \mathcal{H} \hookrightarrow \mathcal{H}_{-1} \\
& \hookrightarrow \cdots \hookrightarrow \mathcal{H}_{-n} \hookrightarrow \mathcal{H}_{-(n+1)} \hookrightarrow \cdots
\end{aligned}
$$

that yields, by an inductive limit and, respectively, a projective limit, a rigged Hilbert space $\mathcal{S} \hookrightarrow \mathcal{H} \hookrightarrow \mathcal{S}^{*}$. In order to make such a construction, the positive selfadjoint operator, that we call the Hamiltonian of the system, and to which this construction is applied, should have a bounded inverse. In case 0 is yet not an eigenvalue of the Hamiltonian but its spectrum accumlates to 0 , this construction fails because the inverse operator, that we call the kernel operator, is no longer bounded and hence, it does not produce continuous embeddings anymore.

In [12] we extended the notion of continuous embedding of Hilbert spaces in the following way. Let $\mathcal{H}$ and $\mathcal{H}_{+}$be two Hilbert spaces. The Hilbert space $\mathcal{H}_{+}$is called closely embedded in $\mathcal{H}$ if:

(ceh1) There exists a linear manifold $\mathcal{D} \subseteq \mathcal{H}_{+} \cap \mathcal{H}$ that is dense in $\mathcal{H}_{+}$.

(ceh2) The embedding operator $j_{+}$with domain $\mathcal{D}$ is closed, as an operator $\mathcal{H}_{+} \rightarrow \mathcal{H}$.

The meaning of the axiom (ceh1) is that on $\mathcal{D}$ the algebraic structures of $\mathcal{H}_{+}$and $\mathcal{H}$ agree. Also, recall that in case $\mathcal{H}_{+} \subseteq \mathcal{H}$ and the embedding operator $j_{+}: \mathcal{H}_{+} \rightarrow \mathcal{H}$ is continuous, one says that $\mathcal{H}_{+}$is continuously embedded in $\mathcal{H}$. 
The operator $A=j_{+} j_{+}^{*}$ is called the kernel operator of the closely embedded Hilbert space $\mathcal{H}_{+}$with respect to $\mathcal{H}$, cf. Schwartz [23]. The model for closely embedded Hilbert spaces follows the results on the Hilbert space $\mathcal{R}(T)$, as defined in [12].

We now extend the notion of triplet of Hilbert spaces to that of triplet of closely embedded Hilbert spaces. By definition, $\left(\mathcal{H}_{+} ; \mathcal{H}^{\prime} \mathcal{H}_{-}\right)$is called a triplet of closely embedded Hilbert spaces if:

(th1) $\mathcal{H}_{+}$is a Hilbert space closely embedded in the Hilbert space $\mathcal{H}$, with the closed embedding denoted by $j_{+}$, and such that $\operatorname{Ran}\left(j_{+}\right)$is dense in $\mathcal{H}$.

(th2) $\mathcal{H}$ is closely embedded in the Hilbert space $\mathcal{H}_{-}$, with the closed embedding denoted by $j_{-}$, and such that $\operatorname{Ran}\left(j_{-}\right)$is dense in $\mathcal{H}_{-}$.

(th3) $\operatorname{Dom}\left(j_{+}^{*}\right) \subseteq \operatorname{Dom}\left(j_{-}\right)$and for every vector $y \in \operatorname{Dom}\left(j_{-}\right) \subseteq \mathcal{H}$ we have

$$
\|y\|_{-}=\sup \left\{\frac{\left|\langle x, y\rangle_{\mathcal{H}}\right|}{\|x\|_{+}} \mid x \in \operatorname{Dom}\left(j_{+}\right), x \neq 0\right\} .
$$

Given a triplet $\left(\mathcal{H}_{+} ; \mathcal{H} ; \mathcal{H}_{-}\right)$of closely embedded Hilbert spaces, and letting $j_{ \pm}$ denote the corresponding closed embeddings of $\mathcal{H}_{+}$in $\mathcal{H}$ and, respectively, of $\mathcal{H}$ in $\mathcal{H}_{-}$, the kernel operator $A=j_{+} j_{+}^{*}$ is a positive selfadjoint operator in $\mathcal{H}$ that is one-to-one. Then, $H=A^{-1}$ is a positive selfadjoint operator in $\mathcal{H}$ and it is called the Hamiltonian of the triplet. Note that, as a consequence of (th3), we actually have $\operatorname{Dom}\left(j_{+}^{*}\right)=\operatorname{Dom}\left(j_{-}\right)$.

In this article we exemplify the abstract notion of triplets of closely embedded Hilbert spaces by a class of spaces of holomorphic functions on the unit polydisc that are called Dirichlet type spaces. The class of Dirichlet type spaces, see Sect. 3 for definition, has been introduced and studied in connection to multipliers' theory by Taylor [24], for the one dimensional case, and by Jupiter and Redett [16], for the multidimensional case. In this respect we note that the notion of " $H^{2}$ duality", that is well-known in the theory of spaces of analytic functions, is close to the notion of triplet of Hilbert spaces when the middle space is the Hardy space $H^{2}$, e.g. the $H^{2}$ duality of Dirchlet and Bergman spaces as in Axler [4], the $H^{2}$ duality of the weighted Bergman space and the Besov space as in Khrushchev and Peller [18], or the general $H^{2}$ duality for Banach spaces of analytic functions as in Aleman, Richter, and Ross [1]. In the case of the multidimensional Dirichlet type spaces on the unit polydisc the weights can have different asymptotics on different directions and hence the classical continuous embeddings may not apply. Our point is that, the closed embeddings can always do the job, with almost no loss, of comparing any pair of Dirichlet type spaces, and that triplets of closely embedded Hilbert spaces and the underlying kernel operators, Hamiltonian operators, and the like, can be naturally associated and explicitly described.

The main result of this article is Theorem 3.1 showing that for any multi-indices $\alpha$ and $\beta$, the Dirichlet type spaces $\mathcal{D}_{\alpha}$ and $\mathcal{D}_{\beta}$ yield a triplet of closely embedded Hilbert spaces $\left(\mathcal{H}_{+} ; \mathcal{H} ; \mathcal{H}_{-}\right)$with $\mathcal{H}_{+}=\mathcal{D}_{\beta}, \mathcal{H}=\mathcal{D}_{\alpha}$, and $\mathcal{H}_{-}=\mathcal{D}_{2 \alpha-\beta}$, the kernel operator $A=j_{+} j_{+}^{*}$ of this triplet is an operator of integral type with kernel provided by the reproducing kernel of the corresponding Dirichlet type Hilbert space and, more generally, both the Hamiltonian $H=A^{-1}$ and the kernel operator $A$ are special cases of radial derivative operators in the sense of Beatrous, Burbea [5]. The technicalities 
of this result are treated in Sect. 2, in the more abstract setting of triplets of Hilbert spaces generated by weighted $L^{2}$ spaces, cf. Theorem 2.1 and its Corollary 2.2. In Sect. 4 we consider probably the most important case, when the middle space is $\mathcal{D}_{0}$, which coincides with the Hardy space $H^{2}$ of the polydisc. In this particular case, the kernel operator is an integral operator defined by the reproducing kernel and we relate the spaces with the classical Dirichlet spaces and their conjugate dual spaces with the Bergman type spaces, see Proposition 4.1. We also make explicit the special cases of $\alpha>0, \alpha<0$, and $N=1$, when even more explicit formulas can be obtained, and the relation to the classical Dirichlet and Bergman space can be clarified as well. As a by-product, we briefly describe a rigging of the Hardy space $H^{2}\left(\mathbb{D}^{N}\right)$ through a scale of Dirichlet type spaces.

Throughout this article we use the terminology and basic facts of operator theory in Hilbert spaces as can be found, e.g. in the monographs of Berezansky, Sheftel, Us [8], Birman and Solomjak [9], or Kato [20].

\section{Triplets of $L^{2}$ Spaces}

Let $(X ; \mathfrak{A})$ be a measurable space on which we consider a $\sigma$-finite measure $\mu$. A function $\omega$ defined on $X$ is called a weight with respect to the measure space $(X ; \mathfrak{A} ; \mu)$ if it is measurable and $0<\omega(x)<\infty$, for $\mu$-almost all $x \in X$. We observe that $\mathcal{W}(X ; \mu)$, the collection of weights with respect to $(X ; \mathfrak{A} ; \mu)$, is a multiplicative unital group. For an arbitrary $\omega \in \mathcal{W}(X ; \mu)$, consider the measure $v$ whose Radon-Nikodym derivative with respect to $\mu$ is $\omega$, denoted $\mathrm{d} \nu=\omega \mathrm{d} \mu$, that is, for any $E \in \mathfrak{A}$ we have $v(E)=\int_{E} \omega \mathrm{d} \mu$. We claim that $v$ is always $\sigma$-finite. Indeed, since $\mu$ is $\sigma$-finite, there exists a sequence $\left(X_{n}\right)_{n}$ of subsets in $\mathfrak{A}$, such that $X_{n} \subseteq X_{n+1}$ and $\mu\left(X_{n}\right)<\infty$ for all $n \geq 1$, with $X=\bigcup_{n \geq 1} X_{n}$. We consider the sequence of measurable subsets of $X$

$$
A_{n}=\left\{x \in X_{n} \mid \omega(x) \leq n\right\}, \quad n \geq 1,
$$

and note that $A_{n} \subseteq A_{n+1}$ for all $n \geq 1$ and that $X \backslash \bigcup_{n \geq 1} A_{n}$ is a measurable set of $\mu$-measure zero. Also,

$$
v\left(A_{n}\right)=\int_{A_{n}} \omega \mathrm{d} \mu \leq n \mu\left(A_{n}\right) \leq n \mu\left(X_{n}\right)<\infty, \quad n \geq 1,
$$

hence $v$ is $\sigma$-finite.

Also, with the notation as before, recall that the Hilbert space $L^{2}(X ; v)$ can be viewed as the weighted space $L_{\omega}^{2}(X ; \mu)$ of all measurable functions $f: X \rightarrow \mathbb{C}$, modulo identification $\mu$-a.e. on $X$, such that

$$
\|f\|_{2, \omega}=\left(\int_{X} \omega|f|^{2} \mathrm{~d} \mu\right)^{1 / 2}<\infty
$$


and naturally organized as a Hilbert space with respect to the norm $\|\cdot\|_{2, \omega}$. We consider the vector space

$$
\mathcal{S}=L^{2}(X ; \mu) \cap L^{2}(X ; \nu)=L^{2}(X ; \mu) \cap L_{\omega}^{2}(X ; \mu) .
$$

In the following we prove that $\mathcal{S}$ is dense in both $L^{2}(X ; \mu)$ and $L^{2}(X ; v)$.

Let $f \in L^{2}(X ; \mu)$ be arbitrary and, for any natural number $n$ let $f_{n}=f \chi_{A_{n}}$, where $A_{n}$ are defined at (2.1). Clearly, $f_{n} \in L^{2}(X ; \mu)$ for all $n \geq 1$. In addition, note that

$$
\int_{X}\left|f_{n}\right|^{2} \mathrm{~d} \nu=\int_{X}|f|^{2} \chi_{A_{n}} \omega \mathrm{d} \mu \leq n \int_{X}\left|f_{n}\right|^{2} \mathrm{~d} \mu<\infty
$$

hence $f_{n} \in L^{2}(X ; v)$ for all $n \geq 1$. On the other hand, the sequence $\left(A_{n}\right)_{n \geq 1}$ is nondecreasing and $X \backslash \cup_{n \geq 1} A_{n}$ has $\mu$-measure zero, hence $f_{n} \rightarrow f \mu$-a.e. on $X$. Since,

$$
\left|f_{n}-f\right|=\chi_{X \backslash A_{n}}|f| \leq|f|, \quad n \geq 1,
$$

we can apply the Dominated Convergence Theorem and conclude that $\left\|f_{n}-f\right\|_{2, \mu} \rightarrow$ 0 , where $\|\cdot\|_{2, \mu}$ denotes the norm of $L^{2}(X ; \mu)$. This shows that $\mathcal{S}$ is dense in $L^{2}(X ; \mu)$.

In order to prove that $\mathcal{S}$ is dense in $L^{2}(X ; v)$ as well, let $g \in L^{2}(X ; v)$ be arbitrary, and define $g_{n}=g \chi_{B_{n}}$, where

$$
B_{n}=\left\{x \in X \mid \frac{1}{n} \leq \omega(x)\right\}, \quad n \geq 1 .
$$

Clearly, $g_{n} \in L^{2}(X ; v)$ for all $n \geq 1$. Also, $\left(B_{n}\right)_{n \geq 1}$ is a nondecreasing sequence of sets in $\mathfrak{A}$ and, due to the assumption that $\omega>0 \mu$-a.e. on $X$, it follows that $X \backslash \bigcup_{n \geq 1} B_{n}$ is a measurable set of $\mu$-measure zero, hence $g_{n} \rightarrow g \mu$-a.e. on X. Since

$$
\int_{X}\left|g_{n}\right|^{2} \mathrm{~d} \mu=\int_{X}|g|^{2} \chi_{B_{n}} \mathrm{~d} \mu \leq n \int_{X}|g|^{2} \chi_{B_{n}} \omega \mathrm{d} \mu \leq n \int_{X}|g|^{2} \mathrm{~d} \nu<\infty,
$$

it follows that $g_{n} \in L^{2}(X ; \mu)$, hence $g_{n} \in \mathcal{S}$ for all $n \geq 1$. On the other hand, $\left|g_{n}-g\right|=$ $\chi_{X \backslash B_{n}}|g| \leq|g|$ for all $n \geq 1$. Thus, we can apply the Dominated Convergence Theorem and conclude that $\left\|g_{n}-g\right\|_{2, v} \rightarrow 0$ as $n \rightarrow \infty$, where $\|\cdot\|_{2, v}$ denotes the norm in $L^{2}(X ; v)$. We have proven that $\mathcal{S}$ is dense in $L^{2}(X ; \nu)$ as well.

Further on, we consider the operator $j_{+}: \mathcal{S}\left(\subseteq L^{2}(X ; \nu)\right) \rightarrow L^{2}(X ; \mu)$, where $\operatorname{Dom}\left(j_{+}\right)=\mathcal{S}$ is defined at (2.2), and $j_{+} f=f$ for all $f \in \operatorname{Dom}\left(j_{+}\right)$. Thus, $j_{+}$is densely defined and has dense range. In the following we prove that $j_{+}$is a closed operator. In order to see this, let $\left(f_{n}\right)$ be a sequence of functions in $\operatorname{Dom}\left(j_{+}\right)=\mathcal{S}$ such that $\left\|f_{n}-f\right\|_{2, v}$ and $\left\|f_{n}-g\right\|_{2, \mu}$ as $n \rightarrow \infty$. For any $m \in \mathbb{N}$ consider the set 
$B_{m}$ as defined in (2.3) and note that

$$
\begin{aligned}
\int_{B_{m}}\left|f_{n}-f\right|^{2} \mathrm{~d} \mu & \leq m \int_{B_{m}}\left|f_{n}-f\right|^{2} \omega \mathrm{d} \mu=m \int_{B_{m}}\left|f_{n}-f\right|^{2} \mathrm{~d} \nu \\
& \leq \int_{X}\left|f_{n}-f\right|^{2} \mathrm{~d} \nu \rightarrow 0, n \rightarrow \infty,
\end{aligned}
$$

hence $f=g \mu$-a.e. on $B_{m}$ and then, taking into account that $X \backslash \bigcup_{m \geq 1} B_{m}$ is a set of $\mu$-measure zero, it follows that $f=g \mu$-a.e. on $X$. Thus, $g=j_{+} f$, and we conclude that the operator $j_{+}$is closed.

In order to determine explicitly the adjoint operator $j_{+}^{*}$, recall that, taking into account the definition of $j_{+}, \operatorname{Dom}\left(j_{+}^{*}\right)$ consists of all $f \in L^{2}(X ; \mu)$ for which there exists $g \in L^{2}(X ; v)$ with the property

$$
\int_{X} u \bar{f} \mathrm{~d} \mu=\int_{X} u \bar{g} \mathrm{~d} \nu=\int_{X} u \bar{g} \omega \mathrm{d} \mu, \text { for all } u \in \operatorname{Dom}\left(j_{+}\right),
$$

and, in this case, $j_{+}^{*} f=g$. After a moment of thought we conclude that $f=\omega g$ $\mu$-a.e. on $X$, equivalently, $g=\omega^{-1} f \mu$-a.e. on $X$. Thus,

$$
\operatorname{Dom}\left(j_{+}^{*}\right)=\left\{f \in L^{2}(X ; \mu) \mid \omega^{-1} f \in L_{\omega}^{2}(X ; \mu)\right\}=L^{2}(X ; \mu) \cap L_{\omega^{-1}}^{2}(X ; \mu),
$$

and $j_{+}^{*} f=\omega^{-1} f$ for all $f \in \operatorname{Dom}\left(j_{+}^{*}\right)$. Also, note that the closed densely defined operator $j_{+}^{*}$ from $L^{2}(X ; \mu)$ in $L^{2}(X ; v)$ is one-to-one, since $\operatorname{Ran}\left(j_{+}\right)=\mathcal{S}$ is dense in $L^{2}(X ; \mu)$.

Further on, denote

$$
\mathcal{H}_{+}=L^{2}(X ; \nu)=L_{\omega}^{2}(X ; \mu), \quad \mathcal{H}=L^{2}(X ; \mu),
$$

and note that, so far we proved that $\mathcal{H}_{+}$is closely embedded in $\mathcal{H}$, with the closed embedding $j_{+}: L^{2}(X ; \mu) \cap L_{\omega}^{2}(X ; \mu)\left(\subseteq L_{\omega}^{2}(X ; \mu)\right) \rightarrow L^{2}(X ; \mu)$, and such that $\operatorname{Ran}\left(j_{+}\right)=\operatorname{Dom}\left(j_{+}\right)=\mathcal{S}$ is dense in $\mathcal{H}$. In the following we define a third Hilbert space $\mathcal{H}_{-}$in such a way that $\left(\mathcal{H}_{+} ; \mathcal{H}^{\prime} \mathcal{H}_{-}\right)$will be a triplet of closely embedded Hilbert spaces, in the sense of the definitions (th1)-(th3) in the Introduction. To this end, on $\operatorname{Dom}\left(j_{+}^{*}\right)$ we define an inner product $\langle\cdot, \cdot\rangle_{-}$by

$$
\langle f, g\rangle_{-}=\left\langle j_{+}^{*} f, j_{+}^{*} g\right\rangle_{L^{2}(X ; v)}, \quad f, g \in \operatorname{Dom}\left(j_{+}^{*}\right)
$$

more precisely,

$$
\langle f, g\rangle_{-}=\int_{X} \omega^{-1} f \overline{\omega^{-1} g} \mathrm{~d} \nu=\int_{X} \omega^{-1} f \bar{g} \mathrm{~d} \mu=\langle f, g\rangle_{L_{\omega^{-1}}^{2}(X ; \mu)}, f, g \in \operatorname{Dom}\left(j_{+}^{*}\right),
$$


with the remark that $\operatorname{Dom}\left(j_{+}^{*}\right)$ is a subspace of $L_{\omega^{-1}}^{2}(X ; \mu)$.

Recall that the measure space $\left(X ; \mathfrak{A} ; \omega^{-1} \mu\right)$ is $\sigma$-finite as well, since $\omega^{-1} \in$ $\mathcal{W}(X ; \mu)$. Note that the measure $\mu$ is absolutely continuous with respect to the measure $\omega^{-1} \mu$ and the Radon-Nikodym derivative $\mathrm{d} \mu / \mathrm{d} \omega^{-1} \mu$ is $\omega$. Thus, we can prove, in a perfectly similar fashion to the proof of the fact that $L^{2}(X ; \mu) \cap L_{\omega}^{2}(X ; \mu)$ is dense in $L^{2}(X ; \mu)$, that $\operatorname{Dom}\left(j_{+}^{*}\right)=L^{2}(X ; \mu) \cap L_{\omega^{-1}}^{2}(X ; \mu)$ is dense in both $L^{2}(X ; \mu)$ and $L_{\omega^{-1}}^{2}(X ; \mu)$.

We let $\mathcal{H}_{-}=L_{\omega^{-1}}^{2}(X ; \mu)$ and also let $j_{-}$be the operator with $\operatorname{Dom}\left(j_{-}\right)=$ $\operatorname{Dom}\left(j_{+}^{*}\right) \subseteq L^{2}(X ; \mu)$ and valued in $L_{\omega^{-1}}^{2}(X ; \mu)$, defined by $j_{-} f=f$ for all $f \in \operatorname{Dom}\left(j_{-}\right)$. In a similar fashion as before, we can show that $j_{-}$is a closed embedding. In addition, $\operatorname{since} \operatorname{Ran}\left(j_{-}\right)=\operatorname{Dom}\left(j_{+}^{*}\right)$, it follows that $j_{-}$has dense range in $\mathcal{H}_{-}$.

We now show that, for any $g \in \operatorname{Dom}\left(j_{-}\right)=\operatorname{Dom}\left(j_{+}^{*}\right)$, we have

$$
\|g\|_{-}=\sup \left\{\frac{\left|\langle f, g\rangle_{\mathcal{H}}\right|}{\|f\|_{+}} \mid f \in \operatorname{Dom}\left(j_{+}\right)\right\} .
$$

Indeed, we consider the linear functional $l_{g}: \mathcal{S} \rightarrow \mathbb{C}$ defined by

$$
l_{g}(f)=\left\langle j_{+} u, f\right\rangle_{\mathcal{H}}, \quad f \in \mathcal{S}=\operatorname{Dom}\left(j_{+}\right),
$$

and note that $l_{g}$ is bounded, with respect to the norm $\|\cdot\|_{+}$, since $g \in \operatorname{Dom}\left(j_{+}^{*}\right)$. Thus, $l_{g}$ can be uniquely extended as a bounded linear functional $\widetilde{l}_{g}: \mathcal{H}_{+} \rightarrow \mathbb{C}$ and there exists uniquely $v=j_{+}^{*} g \in \mathcal{H}_{+}$such that $\widetilde{l}_{g}(f)=\langle f, v\rangle_{+}$, for all $f \in \mathcal{H}_{+}$. In addition, we have

$$
\|v\|_{+}=\left\|j_{+}^{*} g\right\|_{+}=\left\|\widetilde{l}_{g}\right\|_{\mathcal{H}_{+}^{\prime}}=\sup _{0 \neq f \in \mathcal{S}} \frac{\left|\left\langle j_{+} f, g\right\rangle_{\mathcal{H}}\right|}{\|f\|_{+}}
$$

where $\mathcal{H}_{+}^{\prime}$ denotes the topological dual space of $\mathcal{H}_{+}$. Taking into account that, by definition, $\|g\|_{-}=\left\|j_{+}^{*} g\right\|_{+}$, the formula (2.7) follows.

The kernel operator $A=j_{+} j_{+}^{*}$ of the triplet $\left(\mathcal{H}_{+} ; \mathcal{H} ; \mathcal{H}_{-}\right)$is a positive selfadjoint operator in the space $\mathcal{H}=L^{2}(X ; \mu)$ with

$$
\begin{aligned}
\operatorname{Dom}(A) & =\left\{f \in L^{2}(X ; \mu) \mid \omega^{-1} f \in L^{2}(X ; \mu) \cap L^{2}(X ; \nu)\right\} \\
& =L^{2}(X ; \mu) \cap L_{\omega^{-1}}^{2}(X ; \mu) \cap L_{\omega^{-2}}^{2}(X ; \mu),
\end{aligned}
$$

and $A f=\omega^{-1} f$ for all $f \in \operatorname{Dom}(A)$. The Hamiltonian $H=A^{-1}$ is a positive selfadjoint operator in $L^{2}(X ; \mu)$ with

$$
\begin{aligned}
\operatorname{Dom}(H) & =\left\{f \in L^{2}(X ; \mu) \mid \omega f \in L^{2}(X ; \mu)\right\} \\
& =L^{2}(X ; \mu) \cap L_{\omega}^{2}(X ; \mu) \cap L_{\omega^{2}}^{2}(X ; \mu),
\end{aligned}
$$

and $H f=\omega f$ for all $f \in \operatorname{Dom}(H)$. 
Finally, let us observe that $\operatorname{Dom}(H)$ is a subspace of $\mathcal{H}_{+}=L_{\omega}^{2}(X ; \mu)$ and $\operatorname{Ran}(H)$ is a subspace of $\mathcal{H}_{-}=L_{\omega^{-1}}^{2}(X ; \mu)$, hence $H$ can be viewed as a linear operator acting from $\mathcal{H}_{+}$and valued in $\mathcal{H}_{-}$. In this respect, and taking into account the corresponding norms, note that

$$
\|H f\|_{2, \omega^{-1}}^{2}=\int_{X} \omega^{-1}|\omega f|^{2} \mathrm{~d} \mu=\int_{X} \omega|f|^{2} \mathrm{~d} \mu=\|f\|_{2, \omega}, \quad f \in \operatorname{Dom}(H),
$$

that is, $H$ is isometric, when viewed as an operator from $\mathcal{H}_{+}$and valued in $\mathcal{H}_{-}$. It can be proven that $\operatorname{Dom}(H)$ is dense in $\mathcal{H}_{+}$and that $\operatorname{Ran}(H)$ is dense in $\mathcal{H}_{-}$, hence $H$ can be uniquely extended to a unitary operator $\widetilde{H}: \mathcal{H}_{+} \rightarrow \mathcal{H}_{-}$. In a similar way, the kernel operator $A=j_{+} j_{+}^{*}$, when viewed as a linear operator acting from $\mathcal{H}_{-}$and valued in $\mathcal{H}_{+}$, is uniquely extended to a unitary operator $\widetilde{A}: \mathcal{H}_{-} \rightarrow \mathcal{H}_{+}$. In addition, $\widetilde{H}=\widetilde{A}^{-1}$. Then, letting $\mathcal{H}_{+}^{*}$ denote the conjugate dual space of $\mathcal{H}_{+}$, that is, the space of bounded conjugate linear functionals on $\mathcal{H}_{+}$organized as a Hilbert space in a canonical fashion, we can define an operator $\Theta: \mathcal{H}_{-} \rightarrow \mathcal{H}_{+}^{*}$ by

$$
(\Theta g)(f):=\langle\widetilde{A} f, g\rangle_{+}=\int_{X} f \bar{g} \mathrm{~d} \mu, \quad f \in \mathcal{H}_{+}, g \in \mathcal{H}_{-},
$$

and it is easy to see that $\Theta$ is unitary. $\Theta$ is called the canonical unitary identification of $\mathcal{H}_{-}$with the conjugate dual space of $\mathcal{H}_{+}$.

We summarize the facts proved so far in the following theorem. Recall that given a measurable function $\varphi: X \rightarrow \mathbb{C}$, the $\mu$-essential range of $\varphi$, denoted ess $\operatorname{ran}(\varphi)$ is, by definition, the set of all complex numbers $\lambda$ with the property that, for any $\epsilon>0$, $\mu(\{x \in X|| \varphi(x)-\lambda \mid<\epsilon\})>0$. Also, for a linear operator $T$ acting in a Hilbert space, we denote its spectrum by $\sigma(T)$.

Theorem 2.1 Let $\omega$ be a weight on the $\sigma$-finite measure space $(X ; \mathfrak{A} ; \mu)$. Let $\mathcal{H}_{0}=$ $L^{2}(X ; \mu), \mathcal{H}_{+}=L_{\omega}^{2}(X ; \mu)$ and $\mathcal{H}_{-}=L_{\omega^{-1}}^{2}(X ; \mu)$. Then $\left(\mathcal{H}_{+} ; \mathcal{H}_{0} ; \mathcal{H}_{-}\right)$is a triplet of closely embedded Hilbert spaces for which:

(a) The closed embeddings $j_{ \pm}$of $\mathcal{H}_{+}$in $\mathcal{H}_{0}$ and of $\mathcal{H}_{0}$ in $\mathcal{H}_{-}$have maximal domains $L_{\omega}^{2}(X ; \mu) \cap L^{2}(X ; \mu)$ and, respectively, $L^{2}(X ; \mu) \cap L_{\omega^{-1}}^{2}(X ; \mu)$.

(b) The adjoint $j_{+}^{*}$ is defined by $j_{+}^{*} h=\omega^{-1} h$ for all $h \in \operatorname{Dom}\left(j_{+}^{*}\right)=L^{2}(X ; \mu) \cap$ $L_{\omega^{-1}}^{2}(X ; \mu)$.

(c) The kernel operator $A=j_{+} j_{+}^{*}$ is defined by $A h=\omega^{-1} h$, for all $h \in \operatorname{Dom}(A)=$ $L^{2}(X ; \mu) \cap L_{\omega^{-1}}^{2}(X ; \mu) \cap L_{\omega^{-2}}^{2}(X ; \mu)$. Moreover, when viewed as an operator defined in $\mathcal{H}_{-}$and valued in $\mathcal{H}_{+}$, A admits a unique unitary extension $\widetilde{A}: \mathcal{H}_{-} \rightarrow$ $\mathcal{H}_{+}$.

(d) The Hamiltonian $H=A^{-1}$ is defined by $H h=\omega h$ for all $h \in \operatorname{Dom}(H)=$ $L^{2}(X ; \mu) \cap L_{\omega}^{2}(X ; \mu) \cap L_{\omega^{2}}^{2}(X ; \mu)$. Moreover, when viewed as an operator defined in $\mathcal{H}_{+}$and valued in $\mathcal{H}_{-}^{\omega^{2}}, H$ can be uniquely extended to a unitary operator $\widetilde{H}=\widetilde{A}^{-1}$. 
(e) The canonical unitary identification of $\mathcal{H}_{+}^{*}$ with $\mathcal{H}_{-}$is the operator $\Theta$ defined at (2.8).

Consequently, $\sigma(A)=$ ess $\operatorname{ran}\left(\omega^{-1}\right)$ and $\sigma(H)=$ ess ran $(\omega)$. In addition, the embeddings $j_{ \pm}$are simultaneously continuous and this happens if and only if ess $\inf _{X} \omega>0$.

In the following statement we point out a triplet of closely embedded Hilbert spaces generated by two weigthed $L^{2}$ spaces, which is actually equivalent with Theorem 2.1. This statement is useful during the next section.

Corollary 2.2 Let $(X ; \mathfrak{A} ; \mu)$ be a $\sigma$-finite measure space and $\omega_{0}$, $\omega$ be two weights on $X$. Let $\mathcal{H}_{0}=L_{\omega_{0}}^{2}(X ; \mu), \mathcal{H}_{+}=L_{\omega}^{2}(X ; \mu)$, and $\mathcal{H}_{-}=L_{\omega_{0}^{2} \omega^{-1}}^{2}(X ; \mu)$ be the corresponding Hilbert spaces of functions $f: X \rightarrow \mathbb{C}$ whose absolute values are square integrable with respect to the weights $\omega$, $\omega_{0}$, and, respectively, $\omega_{0}^{2} \omega^{-1}$.

Then, $\left(\mathcal{H}_{+} ; \mathcal{H}_{0} ; \mathcal{H}_{-}\right)$is a triplet of closely embedded Hilbert spaces for which:

(a) The closed embeddings $j_{ \pm}$of $\mathcal{H}_{+}$in $\mathcal{H}_{0}$ and, of $\mathcal{H}_{0}$ in $\mathcal{H}_{-}$have maximal domains $L_{\omega}^{2}(X ; \mu) \cap L_{\omega_{0}}^{2}(X ; \mu)$ and, respectively, $L_{\omega_{0}}^{2}(X ; \mu) \cap L_{\omega_{0}^{2} \omega^{-1}}^{2}(X ; \mu)$.

(b) The adjoint $j_{+}^{*}$ is defined by $j_{+}^{*} h=\omega_{0} \omega^{-1} h$ for all $h \in \operatorname{Dom}\left(j_{+}^{*}\right)=L_{\omega_{0}}^{2}(X ; \mu) \cap$ $L_{\omega_{0}^{2} \omega^{-1}}^{2}(X ; \mu)$.

(c) The kernel operator $A=j_{+} j_{+}^{*}$, considered as a positive selfadjoint operator in $\mathcal{H}_{0}$, is defined by $A h=\omega_{0} \omega^{-1} h$, for all $h \in \operatorname{Dom}(A)=L_{\omega_{0}}^{2}(X ; \mu) \cap L_{\omega_{0}^{2} \omega^{-1}}^{2}(X ; \mu) \cap$ $L_{\omega_{0}^{3} \omega^{-2}}^{2}(X ; \mu)$. When considered as an operator from $\mathcal{H}_{-}$to $\mathcal{H}_{+}$, A admits a unique extension to a unitary operator $\widetilde{A}: \mathcal{H}_{-} \rightarrow \mathcal{H}_{+}$, more precisely, $\widetilde{A} f=\omega_{0} \omega^{-1} f$, for all $f \in L_{\omega_{0}^{2} \omega^{-1}}^{2}(X ; \mu)$.

(d) The Hamiltonian $H=A^{-1}$, considered as a positive selfadjoint operator in $\mathcal{H}_{0}$, is defined by $H h=\omega \omega_{0}^{-1} h$ for all $h \in \operatorname{Dom}(H)=L_{\omega_{0}}^{2}(X ; \mu) \cap L_{\omega}^{2}(X ; \mu) \cap$ $L_{\omega^{2} \omega_{0}^{-1}}^{2}(X ; \mu)$. When viewed as an operator from $\mathcal{H}_{+}$to $\mathcal{H}_{-}, H$ admits a unique unitary extension $\widetilde{H}: \mathcal{H}_{+} \rightarrow \mathcal{H}_{-}$, more precisely, $\widetilde{H} g=\omega \omega_{0}^{-1} g$, for all $g \in$ $L_{\omega}^{2}(X ; \mu)$.

(e) The canonical unitary identification of $\mathcal{H}_{+}^{*}=L_{\omega}^{2}(X ; \mu)^{*}$ with $\mathcal{H}_{-}=$ $L_{\omega_{0}^{2} \omega^{-1}}^{2}(X ; \mu)$ is the operator $\Theta$ defined by

$$
(\Theta g)(f):=\langle\widetilde{A} f, g\rangle_{+}=\int_{X} \omega_{0} f \bar{g} d \mu, \quad f \in \mathcal{H}_{+}, g \in \mathcal{H}_{-},
$$

Consequently, $\sigma(A)=$ ess $\operatorname{ran}\left(\omega_{0} \omega^{-1}\right)$ and $\sigma(H)=$ ess $\operatorname{ran}\left(\omega \omega_{0}^{-1}\right)$. In addition, the embeddings $j_{ \pm}$are simultaneously continuous and this happens if and only if ess $\inf _{X} \omega_{0} \omega^{-1}>0$.

Proof This is a consequence of Theorem 2.1 once we calculate the corresponding Radon-Nikodym derivative of $\omega \mathrm{d} \mu$ to $\omega_{0} \mathrm{~d} \mu$, which is $\omega \omega_{0}^{-1}$, and the Radon-Nikodym derivative of $\omega_{0}^{2} \omega^{-1} \mathrm{~d} \mu$ to $\omega_{0} \mathrm{~d} \mu$, which is $\omega_{0} \omega^{-1}$. 


\section{Triplets of Dirichlet Type Spaces: The General Case}

Let $N$ be a fixed natural number and consider the unit polydisc $\mathbb{D}^{N}=\mathbb{D} \times \cdots \times \mathbb{D}$, the direct product of $N$ copies of the unit disc $\mathbb{D}=\{z \in \mathbb{C}|| z \mid<1\}$. For any multi-index $k=\left(k_{1}, \ldots, k_{N}\right) \in \mathbb{Z}_{+}^{N}$ and any $z=\left(z_{1}, \ldots, z_{N}\right) \in \mathbb{C}^{N}$ let $z^{k}=z_{1}^{k_{1}} \cdots z_{N}^{k_{N}}$. We also consider $H\left(\mathbb{D}^{N}\right)$ the algebra of functions holomorphic in the polydisc, that is, the collection of all functions $f: \mathbb{D}^{N} \rightarrow \mathbb{C}$ that are holomorphic in each variable, equivalently, there exists $\left(a_{k}\right)_{k \in \mathbb{Z}_{+}^{N}}$ with the property that

$$
f(z)=\sum_{k \in \mathbb{Z}_{+}^{N}} a_{k} z^{k}, \quad z \in \mathbb{D}^{N},
$$

where the series converges absolutely and uniformly on any compact subset in $\mathbb{D}^{N}$.

Let $\alpha \in \mathbb{R}^{N}$ be fixed. Following Taylor [24], for the one dimensional case, and Jupiter and Redett [16], for the multidimensional case, the Dirichlet type space $\mathcal{D}_{\alpha}$ consists, by definition, on all functions $f \in H\left(\mathbb{D}^{N}\right)$ with representation (3.1) subject to the condition

$$
\sum_{k \in \mathbb{Z}_{+}^{N}}(k+1)^{\alpha}\left|a_{k}\right|^{2}<\infty,
$$

where, with an abuse of notation, $(k+1)^{\alpha}=\left(k_{1}+1\right)^{\alpha_{1}} \cdots\left(k_{N}+1\right)^{\alpha_{N}}$. Actually, it is not necessary to assume that $f \in H\left(\mathbb{D}^{N}\right)$ because the condition (3.2) implies that the function $f$ defined as in (3.1) is holomorphic in $\mathbb{D}^{N}$, see Proposition 2.5 in [16]. The linear space $\mathcal{D}_{\alpha}$ is naturally organized as a Hilbert space with inner product $\langle\cdot, \cdot\rangle_{\alpha}$

$$
\langle f, g\rangle_{\alpha}=\sum_{k \in \mathbb{Z}_{+}^{N}}(k+1)^{\alpha} a_{k} \overline{b_{k}},
$$

where $f$ has representation (3.1) and similarly $g(z)=\sum_{k \in \mathbb{Z}_{+}^{N}} b_{k} z^{k}$, for all $z \in \mathbb{D}^{N}$, and norm $\|\cdot\|_{\alpha}$

$$
\|f\|_{\alpha}^{2}=\sum_{k \in \mathbb{Z}_{+}^{N}}(k+1)^{\alpha}\left|a_{k}\right|^{2} .
$$

A slightly different definition for this type of spaces has been introduced by Kaptanoğlu [17].

For any $\alpha \in \mathbb{R}^{N}$, on the polydisc $\mathbb{D}^{N}$ one can define the following kernel

$$
K^{\alpha}(w, z)=\sum_{k \in \mathbb{Z}_{+}^{N}}(k+1)^{-\alpha} \bar{w}^{k} z^{k}, \quad z, w \in \mathbb{D}^{N},
$$

where, for $w=\left(w_{1}, \ldots, w_{N}\right) \in \mathbb{D}^{N}$ one denotes $\bar{w}=\left(\bar{w}_{1}, \ldots, \bar{w}_{N}\right)$, the entry-wise complex conjugate. As usually, we let $K_{w}^{\alpha}=K^{\alpha}(w, \cdot)$. It turns out, as follows from 
Lemma 2.8 and Lemma 2.9 in [16], that $K^{\alpha}$ is the reproducing kernel for the space $\mathcal{D}_{\alpha}$ in the sense that the following two properties hold:

(rk1) $K_{w}^{\alpha} \in \mathcal{D}_{\alpha}$ for all $w \in \mathbb{D}^{N}$.

(rk2) $f(w)=\left\langle f, K_{w}^{\alpha}\right\rangle_{\alpha}$ for all $f \in \mathcal{D}_{\alpha}$ and all $w \in \mathbb{D}^{N}$.

It follows (this is actually a more general statement, e.g. see Aronszajn [3]) that the set $\left\{K_{w}^{\alpha} \mid w \in \mathbb{D}^{N}\right\}$ is total in $\mathcal{D}_{\alpha}$ and that the kernel $K^{\alpha}$ is positive semidefinite,.

A partial order relation $\geq$ on $\mathbb{R}^{N}$ can be defined by $\alpha \geq \beta$ if and only if $\alpha_{j} \geq \beta_{j}$ for all $j=1, \ldots, N$. In addition, $\alpha>\beta$ means $\alpha_{j}>\beta_{j}$ for all $j=1, \ldots, N$.

Let $\mathcal{P}_{N}$ denote the complex vector space of polynomial functions in $N$ complex variables, that is, those functions $f$ that admit a representation (3.1) for which $\left\{a_{k}\right\}_{k \in \mathbb{Z}_{+}^{N}}$ has finite support. We consider now the additive group $\mathbb{R}^{N}$ and a representation $T:: \mathbb{R}^{N} \rightarrow \mathcal{L}\left(\mathcal{P}_{N}\right)$, where $\mathcal{L}\left(\mathcal{P}_{N}\right)$ denotes the algebra of linear maps on the vector space $\mathcal{P}_{N}$, defined by

$$
\left(T_{\alpha} f\right)(z)=\sum_{k \in \mathbb{Z}_{+}^{N}}(k+1)^{\alpha} a_{k} z^{k}, \quad \alpha \in \mathbb{R}^{N}, z \in \mathbb{D}^{N},
$$

where the polynomial $f$ has representation (3.1) and $\left\{a_{k}\right\}_{k \in \mathbb{Z}_{+}^{N}}$ has finite support. The operators $T_{\alpha}$ can be considered as special types of radial derivative operators, cf. Beatrous and Burbea [5].

Theorem 3.1 For any $\alpha, \beta \in \mathbb{R}^{N}$ we can define a triplet of closely embedded Hilbert spaces $\left(\mathcal{D}_{\beta} ; \mathcal{D}_{\alpha} ; \mathcal{D}_{2 \alpha-\beta}\right)$ with the following properties:

(a) The closed embeddings $j_{ \pm}$of $\mathcal{D}_{\beta}$ in $\mathcal{D}_{\alpha}$ and, respectively, of $\mathcal{D}_{\alpha}$ in $\mathcal{D}_{2 \alpha-\beta}$, have maximal domains $\mathcal{D}_{\alpha} \cap \mathcal{D}_{\beta}$ and, respectively, $\mathcal{D}_{\alpha} \cap \mathcal{D}_{2 \alpha-\beta}$.

(b) The adjoint $j_{+}^{*}$ is defined by $j_{+}^{*} f=T_{\alpha-\beta} f$ for all $f \in \operatorname{Dom}\left(j_{+}^{*}\right)=\mathcal{D}_{\alpha} \cap \mathcal{D}_{2 \alpha-\beta}$.

(c) The kernel operator $A=j_{+} j_{+}^{*}$, considered as a positive selfadjoint operator in $\mathcal{D}_{\alpha}$, is defined by $A f=T_{\alpha-\beta}$ f for all $f \in \operatorname{Dom}(A)=\mathcal{D}_{\alpha} \cap \mathcal{D}_{2 \alpha-\beta} \cap \mathcal{D}_{3 \alpha-2 \beta}$ and is an "integral operator with kernel $K^{\beta}$ ", in the sense that, for all $f \in \operatorname{Dom}(A)$, we have

$$
(A f)(z)=\left\langle f, K_{z}^{\beta}\right\rangle_{\alpha}, \quad z \in \mathbb{D}^{N} .
$$

When viewed as an operator from $\mathcal{D}_{2 \alpha-\beta}$ in $\mathcal{D}_{\beta}$, A admits a unique extension to a unitary operator $\tilde{A}: \mathcal{D}_{2 \alpha-\beta} \rightarrow \mathcal{D}_{\beta}$.

(d) The Hamiltonian operator $H=A^{-1}$ is a positive selfadjoint operator in $\mathcal{D}_{\alpha}$ defined by $H f=T_{\beta-\alpha} f$ for all $f \in \operatorname{Dom}(H)=\mathcal{D}_{\alpha} \cap \mathcal{D}_{\beta} \cap \mathcal{D}_{2 \beta-\alpha}$. When viewed as an operator from $\mathcal{D}_{\beta}$ in $\mathcal{D}_{2 \alpha-\beta}, H$ admits a unique extension to a unitary operator $\widetilde{H}: \mathcal{D}_{\beta} \rightarrow \mathcal{D}_{2 \alpha-\beta}$, and $\tilde{H}=\widetilde{A}^{-1}$.

(e) The canonical unitary identification of $\mathcal{D}_{2 \alpha-\beta}$ with $\mathcal{D}_{\beta}^{*}$ is the operator $\Theta$ defined by

$$
(\Theta g) f=\left\langle T_{\alpha-\beta} f, g\right\rangle_{\beta}, \quad f \in \mathcal{D}_{2 \alpha-\beta}, g \in \mathcal{D}_{\beta} .
$$


In addition, $\sigma(A) \backslash\{0\}=\left\{(k+1)^{\alpha-\beta} \mid k \in \mathbb{Z}_{+}^{N}\right\}$ and $\sigma(H) \backslash\{0\}=\left\{(k+1)^{\beta-\alpha} \mid\right.$ $\left.k \in \mathbb{Z}_{+}^{N}\right\}$. Moreover, the two embeddings $j_{ \pm}$are simultaneously continuous and this happens if and only if $\alpha \leq \beta$.

Proof Let $X=\mathbb{Z}_{+}^{N}$, let $\mathfrak{A}=2^{X}$ be the $\sigma$-algebra of all subsets of $X$, and let $\mu$ denote the counting measure on $X$. Clearly, $(X ; \mathfrak{A} ; \mu)$ is a $\sigma$-finite measure space. We define $\omega_{0}, \omega: X \rightarrow(0, \infty)$ by

$$
\omega_{0}(k)=(k+1)^{\alpha}=\left(k_{1}+1\right)^{\alpha_{1}} \cdots\left(k_{N}+1\right)^{\alpha_{N}}, \quad k=\left(k_{1}, \ldots, k_{N}\right) \in \mathbb{Z}_{+}^{N},
$$

and

$$
\omega(k)=(k+1)^{\beta}=\left(k_{1}+1\right)^{\beta_{1}} \cdots\left(k_{N}+1\right)^{\beta_{N}}, \quad k=\left(k_{1}, \ldots, k_{N}\right) \in \mathbb{Z}_{+}^{N},
$$

Clearly, $\omega_{0}, \omega_{0}^{-1}, \omega$, and $\omega^{-1}$ are trivially all measurable. Note that these are special cases of a canonical morphism from the additive group $\mathbb{R}^{N}$ to the multiplicative semigroup of weights on the measure space $(X ; \mathfrak{A} ; \mu)$. This morphism induces canonical unitary identifications of $L_{\omega}^{2}$ with $\mathcal{D}_{\beta}$, of $L_{\omega_{0}}^{2}$ with $\mathcal{D}_{\alpha}$, as well as the like spaces.

With respect to these identifications, the operator of multiplication with $\omega$ is exactly $T_{\beta}$ while the operator of multiplication with $\omega^{-1}$ is exactly $T_{-\beta}$, and the like multiplication operators. Thus, we can apply Corollary 2.2 and get the properties (a)-(e), except 3.7, but this is only a straightforward verification using (3.3), (3.5), and (3.6).

In addition, observe that the $\mu$-essential ranges of $\omega_{0} \omega^{-1}$ and of $\omega \omega_{0}^{-1}$ are, respectively, their ranges, with the possible exception of 0 , hence by the same Corollary $2.2 \sigma(A) \backslash\{0\}=\operatorname{ran}\left(\omega_{0} \omega^{-1}\right)=\left\{(k+1)^{\alpha-\beta} \mid k \in \mathbb{Z}_{+}^{N}\right\}$ and, similarly, $\sigma(H) \backslash\{0\}=\operatorname{ran}(\omega)=\left\{(k+1)^{\beta-\alpha} \mid k \in \mathbb{Z}_{+}^{N}\right\}$. Consequently, the kernel operator $A$ is bounded if and only if its spectrum is bounded, and this is clearly equivalent with $\beta \geq \alpha$. Then, the continuity of the embeddings $j_{ \pm}$is equivalent with that of $A$.

\section{Triplets of Dirichlet Type Spaces: The Hardy Space $H^{2}\left(\mathbb{D}^{N}\right)$}

In the following we further investigate the special case of triplets when the middle space is $\mathcal{D}_{0}$. The notation is the same with that in the previous section. We first recall, following Rudin [21], how the space $\mathcal{D}_{0}$ is naturally identified with the Hardy space $H^{2}\left(\mathbb{D}^{N}\right)$.

Let $\mathbb{T}=\partial \mathbb{D}$ denote the one-dimensional torus and let $\mathbb{T}^{N}=\mathbb{T} \times \cdot \times \mathbb{T}$ be the $N$-dimensional torus, also called the distinguished boundary of the unit polydisc $\mathbb{D}^{N}$ (which is only a subset of $\partial \mathbb{D}^{N}$ ). Also, we consider the product measure $\mathrm{d} m_{N}=$ $\mathrm{d} m_{1} \times \cdots \times \mathrm{d} m_{1}$ on $\mathbb{D}^{N}$, where $\mathrm{d} m_{1}$ denotes the normalized Lebesgue measure on $\mathbb{T}$, and for any function $f \in H\left(\mathbb{D}^{N}\right)$ and $0 \leq r<1$ let $f_{r}(z)=f(r z)$ for $z \in \mathbb{D}^{N}$. Then, by definition, $f \in H\left(\mathbb{D}^{N}\right)$ belongs to $H^{2}\left(\mathbb{D}^{N}\right)$ if and only if

$$
\sup _{0 \leq r<1} \int_{\mathbb{T}^{N}}\left|f_{r}\right|^{2} \mathrm{~d} m_{N}<\infty
$$


and the norm $\|\cdot\|_{0}$ and inner product $\langle\cdot, \cdot\rangle_{0}$ on the Hardy space $H^{2}\left(\mathbb{D}^{N}\right)$ are defined by

$$
\begin{gathered}
\|f\|_{0}^{2}=\sup _{0 \leq r<1} \int_{\mathbb{T}^{N}}\left|f_{r}\right|^{2} \mathrm{~d} m_{N}=\lim _{r \rightarrow 1-} \int_{\mathbb{T}^{N}}\left|f_{r}\right|^{2} \mathrm{~d} m_{N}, \quad f \in H^{2}\left(\mathbb{D}^{N}\right), \\
\langle f, g\rangle_{0}=\lim _{r \rightarrow 1-} \int_{\mathbb{T}^{N}} f_{r} \overline{g_{r}} \mathrm{~d} m_{N}, \quad f, g \in H^{2}\left(\mathbb{D}^{N}\right),
\end{gathered}
$$

where, for consistency we have used the lower index 0 , because it can be easily proven that this norm coincides with the norm $\|\cdot\|_{0}$ with definition as in (3.4) (here 0 is the multi-index with all entries null). Thus, $\mathcal{D}_{0}$ coincides as a Hilbert space with $H^{2}\left(\mathbb{D}^{N}\right)$. In addition, the reproducing kernel $K^{0}$ has a simple representation in this case, namely in the compact form

$$
K^{0}(w, z)=\frac{1}{1-\bar{w}_{1} z_{1}} \cdots \frac{1}{1-\bar{w}_{N} z_{N}}
$$

In view of the proof of Theorem 3.1, we get:

Proposition 4.1 For any $\alpha \in \mathbb{R}^{N}$ we can define a triplet of closely embedded Hilbert spaces $\left(\mathcal{D}_{\alpha} ; H^{2}\left(\mathbb{D}^{N}\right) ; \mathcal{D}_{-\alpha}\right)$ with the following properties:

(a) The closed embeddings $j_{ \pm}$of $\mathcal{D}_{\alpha}$ in $H^{2}\left(\mathbb{D}^{N}\right)$ and, respectively, of $H^{2}\left(\mathbb{D}^{N}\right)$ in $\mathcal{D}_{-\alpha}$, have maximal domains $H^{2}\left(\mathbb{D}^{N}\right) \cap \mathcal{D}_{\alpha}$ and, respectively, $H^{2}\left(\mathbb{D}^{N}\right) \cap \mathcal{D}_{-\alpha}$.

(b) The adjoint $j_{+}^{*}$ is defined by $j_{+}^{*} f=T_{-\alpha}$ f for all $f \in \operatorname{Dom}\left(j_{+}^{*}\right)=H^{2}\left(\mathbb{D}^{N}\right) \cap \mathcal{D}_{-\alpha}$.

(c) The kernel operator $A=j_{+} j_{+}^{*}$, considered as a positive selfadjoint operator in the Hilbert space $H^{2}\left(\mathbb{D}^{N}\right)$, is defined by $A f=T_{-\alpha} f$ for all $f \in \operatorname{Dom}(A)=$ $H^{2}\left(\mathbb{D}^{N}\right) \cap \mathcal{D}_{-\alpha} \cap \mathcal{D}_{-2 \alpha}$ and is an integral operator with kernel $K^{\alpha}$, in the sense that, for all $f \in \operatorname{Dom}(A)$, we have

$$
(A f)(z)=\left\langle f, K_{z}^{\alpha}\right\rangle_{0}=\lim _{r \rightarrow 1-} \int_{\mathbb{T}^{N}} f_{r}(w) K^{\alpha}(r w, z) d m_{N}(w), \quad z \in \mathbb{D}^{N} .
$$

(d) The Hamiltonian operator $H=A^{-1}$ is a positive selfadjoint operator in $H^{2}\left(\mathbb{D}^{N}\right)$ defined by $H f=T_{\alpha} f$ for all $f \in \operatorname{Dom}(H)=H^{2}\left(\mathbb{D}^{N}\right) \cap \mathcal{D}_{\alpha} \cap \mathcal{D}_{2 \alpha}$.

(e) The canonical unitary identification of $\mathcal{D}_{-\alpha}$ with $\mathcal{D}_{\alpha}^{*}$ is defined by

$$
(\Theta g) f=\left\langle T_{-\alpha} f, g\right\rangle_{\alpha}, \quad f \in \mathcal{D}_{-\alpha}, g \in \mathcal{D}_{\alpha} .
$$

In addition, $\sigma(A) \backslash\{0\}=\left\{(k+1)^{-\alpha} \mid k \in \mathbb{Z}_{+}^{N}\right\}$ and $\sigma(H) \backslash\{0\}=\left\{(k+1)^{\alpha} \mid\right.$ $\left.k \in \mathbb{Z}_{+}^{N}\right\}$. Moreover, the two embeddings $j_{ \pm}$are simultaneously continuous and this happens if and only if $\alpha \geq 0$. 
Proof This is a consequence of Theorem 3.1, except the integral formula in 4.1. To see this, fix $j \in \mathbb{Z}_{+}^{N}$ and $0 \leq r<1$, and observe that

$$
\begin{aligned}
\int_{\mathbb{T}^{N}}(r w)^{j} K^{\alpha}(r w, z) \mathrm{d} m_{N}(w) & =\int_{\mathbb{T}^{N}}(r w)^{j} \sum_{k \in \mathbb{Z}_{+}^{N}}(k+1)^{-\alpha}(r \bar{w})^{k} z^{k} \mathrm{~d} m_{N}(w) \\
& \left.=\int_{\mathbb{T}^{N}} \sum_{k \in \mathbb{Z}_{+}^{N}}(k+1)^{-\alpha} r^{j+k} \bar{w}\right)^{k-j} z^{k} \mathrm{~d} m_{N}(w) \\
& =\sum_{k \in \mathbb{Z}_{+}^{N}}(k+1)^{-\alpha} r^{j+k} z^{k} \int_{\mathbb{T}^{N}} \bar{w}^{k-j} \mathrm{~d} m_{N}(w) \\
& =(j+1)^{-\alpha} r^{2 j} z^{j},
\end{aligned}
$$

where the integral commutes with the series because $z \in \mathbb{D}^{N}$ is fixed and the series converges absolutely and uniformly on the compact space $\mathbb{T}^{N}$. Letting $r \rightarrow 1-$, this shows that 4.1 holds for all functions $f(z)=z^{j}$ with $j \in \mathbb{Z}_{+}^{N}$ and all $z \in \mathbb{D}^{N}$ hence, by linearity, it holds for all $f \in \mathcal{P}_{N}$ and all $z \in \mathbb{D}^{N}$ as well. Further, let us note that $\mathcal{P}_{N}$ is a core for the selfadjoint operator $A$, more precisely, for any $f \in \operatorname{Dom}(A)$ with representation as in (3.1), letting $f_{n} \in \mathcal{P}_{N}$ be the truncation of order $n$ of the power series representing $f$,

$$
f_{n}(z)=\sum_{k \in \mathbb{Z}_{+}^{N},|k| \leq n} a_{k} z^{k}, \quad z \in \mathbb{D}^{N}
$$

for arbitrary $n \in \mathbb{N}$, we have $\left\|f_{n}-f\right\|_{0} \rightarrow 0$ and $\left\|A f_{n}-A f\right\|_{0} \rightarrow 0$ as $n \rightarrow \infty$. Consequently,

$$
(A f)(z)=\lim _{n \rightarrow \infty}\left(A f_{n}\right)(z)=\lim _{n \rightarrow \infty}\left\langle f_{n}, K_{z}^{\alpha}\right\rangle_{0}=\left\langle f, K_{z}^{\alpha}\right\rangle_{0}, \quad z \in \mathbb{D}^{N},
$$

hence 4.1 is completely proven.

In case $\alpha \nsupseteq 0$ and $\alpha \not \leq 0$, that means that $\alpha_{j}>0$ and $\alpha_{k}<0$ for some $j, k \in$ $\{1, \ldots, N\}$, hence $\inf \left\{(k+1)^{\alpha} \mid k \in \mathbb{Z}_{+}^{N}\right\}=0$ and $\sup \left\{(k+1)^{\alpha} \mid k \in \mathbb{Z}_{+}^{N}\right\}=+\infty$, from Theorem 3.1 it follows that both the kernel operator $A$ and the Hamiltonian operator $H$ are unbounded. This case shows that the mixed sign case cannot be reduced either directly or dually to the case when the embeddings are continuous. In addition, when considering the embedding $j_{+}$, we have $\operatorname{Dom}\left(j_{+}\right)=\mathcal{D}_{\alpha} \cap H^{2}\left(\mathbb{D}^{N}\right)$ which is a linear manifold strictly contained in $\mathcal{D}_{\alpha}$ and strictly contained in $H^{2}\left(\mathbb{D}^{N}\right)$.

In the following we describe particular cases with respect to the multi-index $\alpha$ in Proposition 4.1, and point out the relations with classical Dirichlet spaces and Bergman spaces. 


\subsection{Case $\alpha \geq 0$.}

Assume that $\alpha_{k} \geq 0$ for all $k=1, \ldots, N$ and that $\alpha_{k}>0$ for some $k \in\{1, \ldots, N\}$, that is, we exclude the trivial case $\alpha=0$. Since $\left(k_{j}+1\right)^{\alpha_{j}} \geq 1$ for all $j=1, \ldots, N$, it follows that $\|f\|_{0} \leq\|f\|_{\alpha}$ for all $f \in H^{2}\left(\mathbb{D}^{N}\right)$, equivalently $\mathcal{D}_{\alpha}$ is contractively embedded in $\mathcal{D}_{0}$. With respect to the associated triplet of Hilbert spaces $\left(\mathcal{D}_{\alpha} ; \mathcal{D}_{0} ; \mathcal{D}_{-\alpha}\right)$ and the notation as in Proposition 4.1, we have $\sigma(A)=\operatorname{ran}\left(\omega^{-1}\right)=\left\{(k+1)^{-\alpha} \mid k \in\right.$ $\left.\mathbb{Z}_{+}^{N}\right\} \cup\{0\}$, the operator $A$ is compact, 0 is not an eigenvalue but it is an accumulation point of the spectrum $\sigma(A)$. Thus, $j_{+}$is a compact embedding of $\mathcal{D}_{\alpha}$ into $\mathcal{D}_{0}$. On the other hand, the kernel operator of the embedding $j_{-}$of $\mathcal{D}_{0}$ into $\mathcal{D}_{-\alpha}$ is unitarily equivalent to $A$, hence $j_{-}$is a compact operator as well.

In addition, the Hamiltonian operator $H$ is a unbounded positive selfadjoint operator with $\sigma(H)=\left\{(k+1)^{\alpha} \mid k \in \mathbb{Z}_{+}^{N}\right\}$, hence it is boundedly invertible, actually the whole interval $(-\infty, 1)$ is in the resolvent set of $H$. As a linear operator in the Hilbert space $\mathcal{D}_{0}, H$ acts as $T_{\alpha}$, see (3.6), with maximal domain $\operatorname{Dom}(H)=\left\{f \in \mathcal{D}_{0} \mid T_{\alpha} f \in \mathcal{D}_{0}\right\}$.

\subsection{Case $\alpha<0$.}

The spaces $\mathcal{D}_{\alpha}$ for $\alpha<0$ are of Bergman type and are of special importance. More precisely, let $\mathrm{d} A_{1}$ denote the normalized area measure on the unit disc $\mathbb{D}$ and let $\mathrm{d} A_{N}=\mathrm{d} A_{1} \times \cdots \times \mathrm{d} A_{1}$ be the corresponding product measure on $\mathbb{D}^{N}$. Then, e.g. see Lemma 2.15 in [16], a function $f \in H\left(\mathbb{D}^{N}\right)$ is in $\mathcal{D}_{\alpha}$, for a fixed $\alpha<0$ in $\mathbb{R}^{N}$, if and only if

$$
\int_{\mathbb{D}^{N}}|f(z)|^{2} \frac{1}{\left(1-\left|z_{1}\right|^{2}\right)^{1+\alpha_{1}}} \cdots \frac{1}{\left(1-\left|z_{N}\right|^{2}\right)^{1+\alpha_{N}}} \mathrm{~d} A_{N}(z)<\infty,
$$

and, in this case, the norm $\|\cdot\|_{\alpha}$ on $\mathcal{D}_{\alpha}$ is equivalent to the norm $\|\cdot\|_{\mathrm{a}, \alpha}$ defined by

$$
\|f\|_{\mathrm{a}, \alpha}^{2}=\int_{\mathbb{D}^{N}}|f(z)|^{2} \frac{1}{\left(1-\left|z_{1}\right|^{2}\right)^{1+\alpha_{1}}} \cdots \frac{1}{\left(1-\left|z_{N}\right|^{2}\right)^{1+\alpha_{N}}} \mathrm{~d} A_{N}(z) .
$$

Let $A_{\alpha}^{2}\left(\mathbb{D}^{N}\right)$ denote this Hilbert space, with norm $\|\cdot\|_{\mathrm{a}, \alpha}$ defined as in (4.2) (so, $A_{\alpha}^{2}\left(\mathbb{D}^{N}\right)=\mathcal{D}_{\alpha}$ as topological spaces, but not isometrically). A calculation similar with the one performed in the proof of Lemma 2.15 in [16] for $N=2$, shows that, for all $f \in A_{\alpha}^{2}\left(\mathbb{D}^{N}\right)$, we have

$$
\|f\|_{\mathrm{a}, \alpha}^{2}=2^{N} \sum_{k \in \mathbb{Z}_{+}^{N}} \frac{1}{-\alpha\left(\begin{array}{c}
k-\alpha \\
-\alpha
\end{array}\right)}\left|a_{k}\right|^{2}, \quad f(z)=\sum_{k \in \mathbb{Z}_{+}^{N}} a_{k} z^{k}, z \in \mathbb{D}^{N},
$$

where we denote $-\alpha\left(\begin{array}{c}k-\alpha \\ -\alpha\end{array}\right)=\left(-\alpha_{1}\right)\left(\begin{array}{c}k_{1}-\alpha_{1} \\ -\alpha_{1}\end{array}\right) \cdots\left(-\alpha_{n}\right)\left(\begin{array}{c}k_{N}-\alpha_{N} \\ -\alpha_{N}\end{array}\right)$ and use the generalized binomial coefficients. From here it follows that there exists $C_{\alpha}>0$ such that $\|f\|_{\mathrm{a}, \alpha} \leq$ $C_{\alpha}\|f\|_{2}$ for all $f \in H^{2}\left(\mathbb{D}^{N}\right)$, hence $H^{2}\left(\mathbb{D}^{N}\right)$ is continuously embedded into $A_{\alpha}^{2}\left(\mathbb{D}^{N}\right)$. 
On the other hand, there is a naturally defined closed embedding of $A_{\alpha}^{2}\left(\mathbb{D}^{N}\right)$ into $H^{2}\left(\mathbb{D}^{N}\right)$, let us denote it by $j_{+}$. Using arguments similar to those in the proof of Theorem 3.1, it follows that $\operatorname{Dom}\left(j_{+}\right)=H^{2}\left(\mathbb{D}^{N}\right)$, when viewed as a linear submanifold of $A_{\alpha}^{2}\left(\mathbb{D}^{N}\right)$, and that the kernel operator of this embedding $A=j_{+} j_{+}^{*}$ is the operator in $H^{2}\left(\mathbb{D}^{N}\right)$ defined by

$$
(A f)(z)=2^{-N} \sum_{k \in \mathbb{Z}_{+}^{N}}-\alpha\left(\begin{array}{c}
-\alpha \\
k-\alpha
\end{array}\right) a_{k} z^{k}, f(z)=\sum_{k \in \mathbb{Z}_{+}^{N}} a_{k} z^{k}, f \in \operatorname{Dom}(A), z \in \mathbb{D}^{N}
$$

with maximal domain $\operatorname{Dom}(A)=\left\{f \in H^{2}\left(\mathbb{D}^{N}\right) \mid A f \in H^{2}\left(\mathbb{D}^{N}\right)\right\}$. Then, the spectrum of $A$ is $\sigma(A)=\left\{-\alpha 2^{-N}\left(\begin{array}{c}k-\alpha \\ -\alpha\end{array}\right) \mid k \in \mathbb{Z}_{+}^{N}\right\}$ and taking into account (e.g. see Hardy [15]) that $\left(\begin{array}{c}-\alpha \\ k-\alpha\end{array}\right)$ is asymptotic to $(k+1)^{-\alpha}$, it follows that the kernel operator $A$ is unbounded, while the Hamiltonian operator $H=A^{-1}$ is compact. This case is dual to that of $\alpha \geq 0$, when the embeddings were continuous, in an obvious fashion.

We consider the triplet of closed embeddings of Hilbert spaces $\left(A_{\alpha}^{2}\left(\mathbb{D}^{N}\right) ; H^{2}\left(\mathbb{D}^{N}\right)\right.$; $\left.\mathcal{H}_{-}\right)$as an extension of the closed embedding $j_{+}$, and want to find a suitable realization for the Hilbert space $\mathcal{H}_{-}$. This can be defined as the space of all functions $f \in H\left(\mathbb{D}^{N}\right)$ such that

$$
\sum_{k \in \mathbb{Z}_{+}^{N}}-\alpha\left(\begin{array}{c}
k-\alpha \\
-\alpha
\end{array}\right)\left|a_{k}\right|^{2}<\infty
$$

with norm $\|\cdot\|_{-}$defined by

$$
\|f\|_{-}^{2}=2^{-N} \sum_{k \in \mathbb{Z}_{+}^{N}}-\alpha\left(\begin{array}{c}
k-\alpha \\
-\alpha
\end{array}\right)\left|a_{k}\right|^{2}, \quad f \in \mathcal{H}_{-}
$$

where for $f$ we have used the Taylor representation (3.1).

\subsection{Case $N=1$ and $\alpha=1$.}

We now specialize even more, letting $N=1$ and $\alpha=1$. The classical Dirichlet space $\mathcal{D}$ is defined as the space of all functions $f$ holomorphic in the open unit disc $\mathbb{D}$ and such that

$$
\|f\|_{\mathrm{d}}^{2}=|f(0)|^{2}+\frac{1}{\pi} \int_{\mathbb{D}}\left|f^{\prime}(z)\right|^{2} \mathrm{~d}(x, y)<\infty, \quad z=x+\mathrm{i} y,
$$

and it follows that $\mathcal{D}$ equals, with our notation, $\mathcal{D}_{1}$, but the two norms do not coincide, they are only equivalent. So, topologically, $\mathcal{D}$ and $\mathcal{D}_{1}$ are the same, but not isometrically. For a recent survey on the classical Dirichlet space and related theory, see Arcozzi, Rochberg, Sawyer, and Wick [2]. If we consider the corresponding triplet 
of Hilbert spaces we get $\left(\mathcal{D} ; H^{2} ; \mathcal{H}_{-}\right)$, where $\mathcal{H}_{-}$coincides, as a topological space, with the Bergman space $A^{2}(\mathbb{D})$, e.g. see Duren and Schuster [11], of all functions $f$ holomorphic in the open unit disc $\mathbb{D}$ and such that

$$
\|f\|_{\mathrm{a}}^{2}=\frac{1}{\pi} \int_{\mathbb{D}}|f(z)|^{2} \mathrm{~d}(x, y)<\infty, \quad z=x+\mathrm{i} y,
$$

which, in terms of Taylor coefficients, is

$$
\|f\|_{\mathrm{a}}^{2}=\sum_{n=0}^{\infty} \frac{\left|a_{n}\right|^{2}}{n+1}, \quad f(z)=\sum_{n=0}^{\infty} a_{n} z^{n}
$$

The corresponding embeddings are compact as well, the kernel operator $A$ is the integral operator

$$
(A f)(z)=f(0)+\int_{0}^{z} \frac{f(w)-f(0)}{w} \mathrm{~d} w, \quad f \in H^{2}(\mathbb{D}), \quad z \in \mathbb{D},
$$

where the integral can be performed on any Jordan curve connecting 0 and $z$ in $\mathbb{D}$. $A$ is a Hilbert-Schmidt operator with spectrum $\sigma(A)=\left\{\frac{1}{n} \mid n \in \mathbb{N}\right\}$, with all eigenvalues simple except the eigenvalue 1 that has multiplicity 2 . The Hamiltonian operator $H$ is a maximal differential operator

$$
(H f)(z)=[z f(z)]^{\prime}=f(z)+z f^{\prime}(z), \quad f \in \operatorname{Dom}(H), z \in \mathbb{D},
$$

where $\operatorname{Dom}(H)=\left\{f \in H^{2}(\mathbb{D}) \mid H f \in H^{2}(\mathbb{D})\right\}$. As before, $H$ is a unbounded positive selfadjoint operator with $\sigma(H)=\mathbb{N}$, and with compact resolvent.

\subsection{Case $N=1$ and $\alpha=-1$.}

For the case $N=1$ and $\alpha=-1$, we get $A_{-1}^{2}(\mathbb{D})=A^{2}(\mathbb{D})$, the classical Bergman space of all functions $f \in H(\mathbb{D})$ that are square integrable on $\mathbb{D}$ with respect to the planar measure, see (4.5) and (4.6). We can produce a triplet of Hilbert spaces $\left(A^{2}(\mathbb{D}) ; H^{2}(\mathbb{D}) ; \mathcal{D}\right)$, where $\mathcal{D}$ is the classical Dirichlet space as defined at $(4.4)$, in the following way. The corresponding kernel operator $A$ of the closed embedding of $A^{2}(\mathbb{D})$ into $H^{2}(\mathbb{D})$ is the maximal differential operator defined by

$$
(A f)(z)=[z f(z)]^{\prime}=f(z)+z f^{\prime}(z), \quad f \in \operatorname{Dom}(A)
$$


which is unbounded, while the Hamiltonian operator $H$ is defined by

$$
(H f)(z)=\frac{1}{z} \int_{0}^{z} f(w) \mathrm{d} w, \quad f \in H^{2}(\mathbb{D}),
$$

and is a Hilbert-Schmidt operator.

\section{Rigging the Hardy Space $H^{2}\left(\mathbb{D}^{N}\right)$ with Dirichlet Type Spaces}

In the following we briefly show that the Hardy space $H^{2}\left(\mathbb{D}^{N}\right)$, identified with the Dirichlet type space $\mathcal{D}_{0}$ as in Sect. 4 , can be rigged in a natural way through a scale of Dirichlet type spaces. The rigging and the scale of Hilbert spaces have to be understood in the classical sense, that is, when all the embeddings are continuous, cf. Gelfand and Vilenkin [14] and, respectively, Berezanskii [6].

We consider all Dirichlet type spaces $\mathcal{D}_{\alpha}\left(\mathbb{D}^{N}\right)$ for $\alpha \geq 0$. By Proposition 4.1, $\mathcal{D}_{\alpha}\left(\mathbb{D}^{N}\right)$ is continuously embedded in $H^{2}\left(\mathbb{D}^{N}\right)=\mathcal{D}_{0}\left(\mathbb{D}^{N}\right)$ for all $\alpha \geq 0$. In addition, if $\alpha \neq 0$ then the embedding $\mathcal{D}_{\alpha}\left(\mathbb{D}^{N}\right) \hookrightarrow H^{2}\left(\mathbb{D}^{N}\right)$ is contractive and compact. In addition, the same is true for the embedding $\mathcal{D}_{\beta}\left(\mathbb{D}^{N}\right) \hookrightarrow \mathcal{D}_{\alpha}\left(\mathbb{D}^{N}\right)$ whenver $\beta \geq \alpha$ and $\beta \neq \alpha$. Therefore, with respect to continuous embeddings, the family $\left\{\mathcal{D}_{\alpha}\left(\mathbb{D}^{N}\right)\right\}_{\alpha \geq 0}$ is a projective system of Hilbert spaces and hence it makes sense to consider its projective limit

$$
\mathcal{S}\left(\mathbb{D}^{N}\right)=\lim _{\alpha \geq 0} \operatorname{proj} \mathcal{D}_{\alpha}\left(\mathbb{D}^{N}\right)
$$

Recall that this means that $\mathcal{S}\left(\mathbb{D}^{N}\right)=\bigcap_{\alpha \geq 0} \mathcal{D}_{\alpha}\left(\mathbb{D}^{N}\right)$, with the locally convex topology defined by the family of quadratic norms $\left\{\|\cdot\| \mid \alpha \in \mathbb{R}^{N}, \alpha \geq 0\right\}$, with norms $\|\cdot\|_{\alpha}$ defined at (3.4).

The locally convex space $\mathcal{S}\left(\mathbb{D}^{N}\right)$ is actually a Fréchet space and the embedding $\mathcal{S}\left(\mathbb{D}^{N}\right) \hookrightarrow H^{2}\left(\mathbb{D}^{N}\right)$ is nuclear. To see this we can construct a scale of continuously embedded Dirichlet type spaces that has the same projective limit and with corresponding nuclear embeddings. More precisely, for each $n \in \mathbb{N}$ let, with an abuse of notation, $\mathcal{D}_{n}$ denote the Dirichlet type space corresponding to the multiindex $(n, \ldots, n) \in \mathbb{N}^{N} \subset \mathbb{R}^{N}$. Because, for each $\alpha \in \mathbb{R}^{N}$ there exists $n \in \mathbb{N}$ with $\alpha \leq(n, \ldots, n)$, it follows that

$$
\mathcal{S}\left(\mathbb{D}^{N}\right)=\lim _{n \in \mathbb{N}} \operatorname{proj} \mathcal{D}_{n},
$$

hence $\mathcal{S}\left(\mathbb{D}^{N}\right)$ is metrizable and then its completeness follows by a standard argument. On the other hand, we actually have a (left) scale of Hilbert spaces

$$
\cdots \hookrightarrow \mathcal{D}_{n+1} \hookrightarrow \mathcal{D}_{n} \hookrightarrow \cdots \mathcal{D}_{1} \hookrightarrow \mathcal{D}_{0}=H^{2}\left(\mathbb{D}^{N}\right)
$$


All the embeddings are continuous, actually compact, and each space in the scale is dense in its successor (and hence in all of its successors). Moreover, for any $n \geq 1$, letting $A$ denote the kernel operator of the embedding $\mathcal{D}_{n+2} \hookrightarrow \mathcal{D}_{n}$, we have $\sigma(A)=$ $\left\{\left(1+k_{1}\right)^{-2} \cdots\left(1+k_{N}\right)^{-2} \mid k \in \mathbb{Z}_{+}^{N}\right\} \cup\{0\}$, hence $A$ is a trace-class (nuclear) operator, which makes the corresponding embedding $\mathcal{D}_{n+2} \hookrightarrow \mathcal{D}_{n}$ to be a Hilbert-Schmidt (quasi-nuclear) operator. Therefore, the projective limit $\mathcal{S}\left(\mathbb{D}^{N}\right)$ is a nuclear Fréchet space. In addition, note that the algebra $\mathcal{P}_{N}$ of polynomials in $N$ indeterminates is contained in $\mathcal{S}\left(\mathbb{D}^{N}\right)$, in particular $\mathcal{S}\left(\mathbb{D}^{N}\right)$ is dense in $H^{2}\left(\mathbb{D}^{N}\right)$. However, $\mathcal{S}\left(\mathbb{D}^{N}\right)$ does not coincide with $\mathcal{P}_{N}$.

In a similar way, the conjugate dual spaces $\mathcal{D}_{-\alpha}=\mathcal{D}_{\alpha}^{*}$, indexed by the directed set $\alpha \geq 0$, make an inductive system of Hilbert spaces and hence it makes sense to consider its inductive limit

$$
\mathcal{S}^{*}\left(\mathbb{D}^{N}\right)=\operatorname{limind}_{\alpha \geq 0} \mathcal{D}_{-\alpha},
$$

where $\mathcal{S}^{*}\left(\mathbb{D}^{N}\right)=\bigcup_{\alpha \geq 0} \mathcal{D}_{-\alpha}$, endowed with the strongest topology that makes all the embeddings $\mathcal{D}_{-\alpha} \hookrightarrow \mathcal{S}^{*}\left(\mathbb{D}^{N}\right)$ continuous. Actually, it is preferable to see that the scale in (5.1) can be fully extended to a scale that produces a rigging of $H^{2}\left(\mathbb{D}^{N}\right)$

$$
\begin{aligned}
\cdots & \hookrightarrow \mathcal{D}_{n+1} \hookrightarrow \mathcal{D}_{n} \hookrightarrow \cdots \mathcal{D}_{1} \hookrightarrow H^{2}\left(\mathbb{D}^{N}\right) \hookrightarrow \mathcal{D}_{-1} \\
\hookrightarrow & \hookrightarrow \hookrightarrow \mathcal{D}_{-n} \hookrightarrow \mathcal{D}_{-n-1} \hookrightarrow \cdots .
\end{aligned}
$$

In particular, $\mathcal{S}^{*}\left(\mathbb{D}^{N}\right)$ can be realized as the inductive limit of the system $\left(\mathcal{D}_{-n}\right)_{n \geq 0}$, where $\mathcal{D}_{-n}=\mathcal{D}_{n}^{*}$ is the Dirichlet type space with index $(-n, \ldots,-n) \in \mathbb{Z}_{-}^{N}$ for all $n \in \mathbb{N}$ and is complete. Note that $H^{2}\left(\mathbb{D}^{N}\right)$ is also dense in $\mathcal{S}^{*}\left(\mathbb{D}^{N}\right)$.

Recalling the notation in Sect. 4.2, it follows that $\mathcal{S}^{*}\left(\mathbb{D}^{N}\right)$ can be realized as the inductive limit of Bergman type spaces $A_{\alpha}^{2}\left(\mathbb{D}^{N}\right)$ for $\alpha<0$,

$$
\mathcal{S}^{*}\left(\mathbb{D}^{N}\right)=\operatorname{limind}_{\alpha<0} A_{\alpha}^{2}\left(\mathbb{D}^{N}\right),
$$

since for all $\alpha<0$ the Dirichlet type space $\mathcal{D}_{\alpha}$ coincides with the Bergman space $A_{\alpha}^{2}\left(\mathbb{D}^{N}\right)$ as topological spaces of holomorphic functions, even though their norms are not identical, but still equivalent.

In conclusion, $\mathcal{S}\left(\mathbb{D}^{N}\right) \hookrightarrow H^{2}\left(\mathbb{D}^{N}\right) \hookrightarrow \mathcal{S}^{*}\left(\mathbb{D}^{N}\right)$ is a rigging of the Hardy space $H^{2}\left(\mathbb{D}^{N}\right)$ obtained through a scale of Dirichlet type spaces as in (5.2).

\section{References}

1. Aleman, A., Richter, S., Ross, W.T.: Pseudocontinuations and the backward shift. Indiana Univ. J. 47, 223-276 (1998)

2. Arcozzi, N., Rochberg, R., Sawyer, E., Wick, B.: The Dirichlet space: a survey. N. Y. J. Math. 17A, 45-86 (2011)

3. Aronszajn, N.: Theory of reproducing kernels. Trans. Amer. Math. Soc. 68, 337-404 (1950)

4. Axler, S.: Bergman spaces and their operators. In: Surveys of Some Recent Results in Operator Theory, vol. I, pp. 1-50. Longman Science and Technology, Harlow (1988) 
5. Beatrous, F., Burbea, J.: Holomorphic Sobolev spaces on the ball. Dissertationes Math. (Rozprawy Mat.) 276, 60 (1989)

6. Berezanskii, Yu.M: Expansions in Eigenfunctions of Selfadjoint Operators. American Mathematical Society, Providence (1968)

7. Berezansky, Yu.M., Kondratiev, Y.G.: Spectral methods in infinite-dimensional analysis. In: Mathematical Physics and Applied Mathematics. Kluwer Academic Publishers, Dordrecht (1995)

8. Berezansky, Yu.M, Sheftel, Z.G., Us, G.F.: Functional Analysis. Vols. I and II. Birkhäuser, Basel (1996)

9. Birman, M.S., Solomjak, M.Z.: Spectral Theory of Self-Adjoint Operators in Hilbert Space. D. Reidel Publishing Company, Dordrecht-Boston-Lancaster-Tokyo (1987)

10. Bohm, A., Gadella, M.: Dirac Kets, Gamow Vectors, and Gel'fand Triplets: the Rigged Hilbert Space Formulation of Quantum Mechanics. In: Lecture Notes in Physics, vol. 348. Springer, Berlin (1989)

11. Duren, P., Schuster, A.: Bergman Spaces, Mathematical Surveys and Monographs. American Mathematical Society, Providence (2004)

12. Cojuhari, P.A., Gheondea, A.: Closed embeddings of Hilbert spaces. J. Math. Anal. Appl. 369, 60-75 (2010)

13. Gadella, M.: A rigged Hilbert space of Hardy-class functions: applications to resonances. J. Math. Phys. 24, 1462-1469 (1983)

14. Gelfand, I.M., Vilenkin, N.Y.: Generalized Functions, vol. IV. Academic Press, New York (1964)

15. Hardy, G.H.: Divergent Series. AMS Chelsea Publishing, New York (2000)

16. Jupiter, D., Redett, D.: Multipliers on Dirichlet type spaces. Acta. Sci. Math. (Szeged) 72, 179-203 (2006)

17. Kaptanoğlu, H.K.: Möbius-invariant Hilbert spaces in polydiscs. Pacific J. Math. 163, 337-360 (1994)

18. Khrushchev, S., Peller, V.: Hankel operators, best approximation, and stationary Gausssian processes. Russian Math. Surveys 37, 61-144 (1982)

19. de la Madrid, R.: Quantum mechanics in Rigged Hilbert Space language. Dissertation, University of Texas at Austin, PhD (2002)

20. Kato, T.: Perturbation Theory for Linear Operators. Springer, Berlin (1966)

21. Rudin, W.: Function Theory in Polydiscs. W.A. Benjamin, New York (1969)

22. Schwartz, L.: Théorie des distributions. Hermann et Cie, Paris (1950)

23. Schwartz, L.: Sous espace Hilbertiens d'espaces vectoriel topologiques et noyaux associés (noyaux reproduisants). J. Anal. Math. 13, 115-256 (1964)

24. Taylor, G.D.: Multipliers on $D_{\alpha}$. Trans. Amer. Math. Soc. 123, 229-240 (1966) 